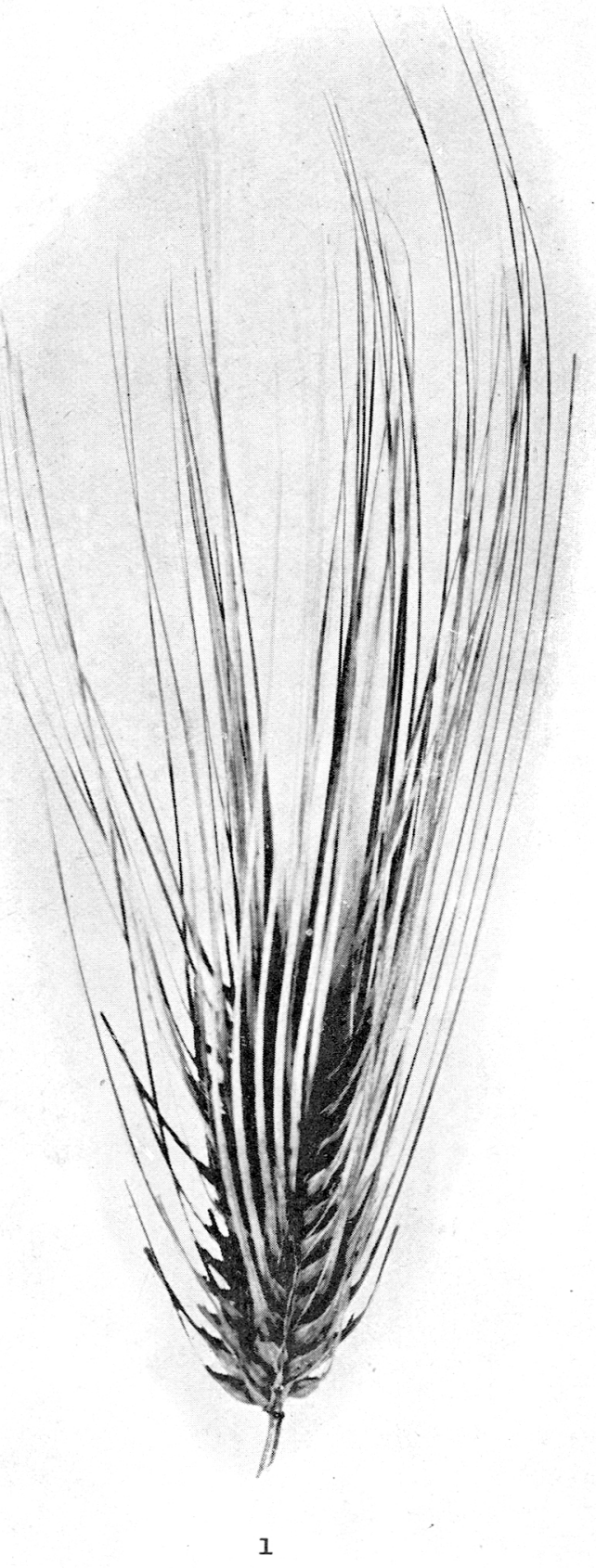

(Form.=A.AEEii)

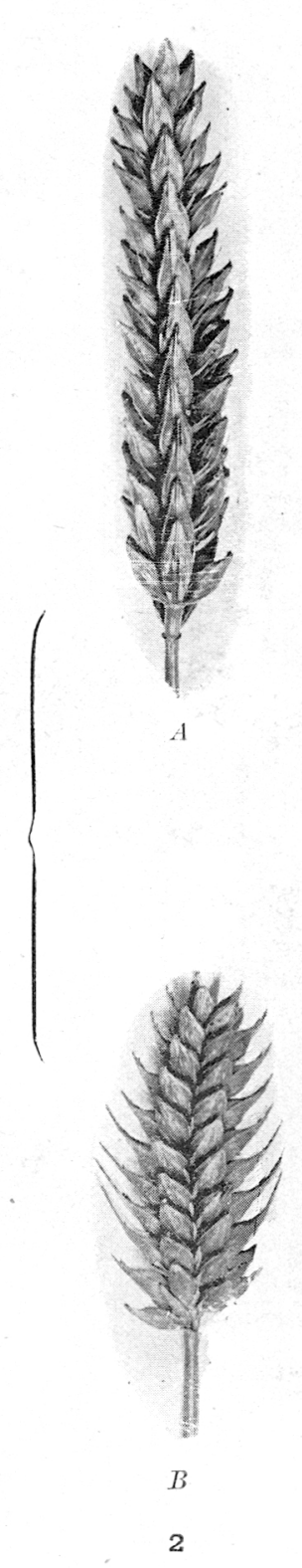

$($ Form. $=$ aaeeII $)$ 


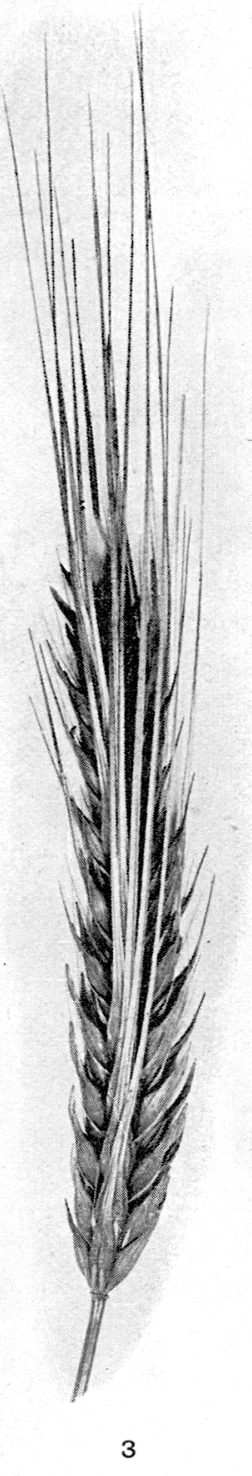

$($ Form $=\mathrm{A} a \mathrm{EeI} \mathbf{i})$

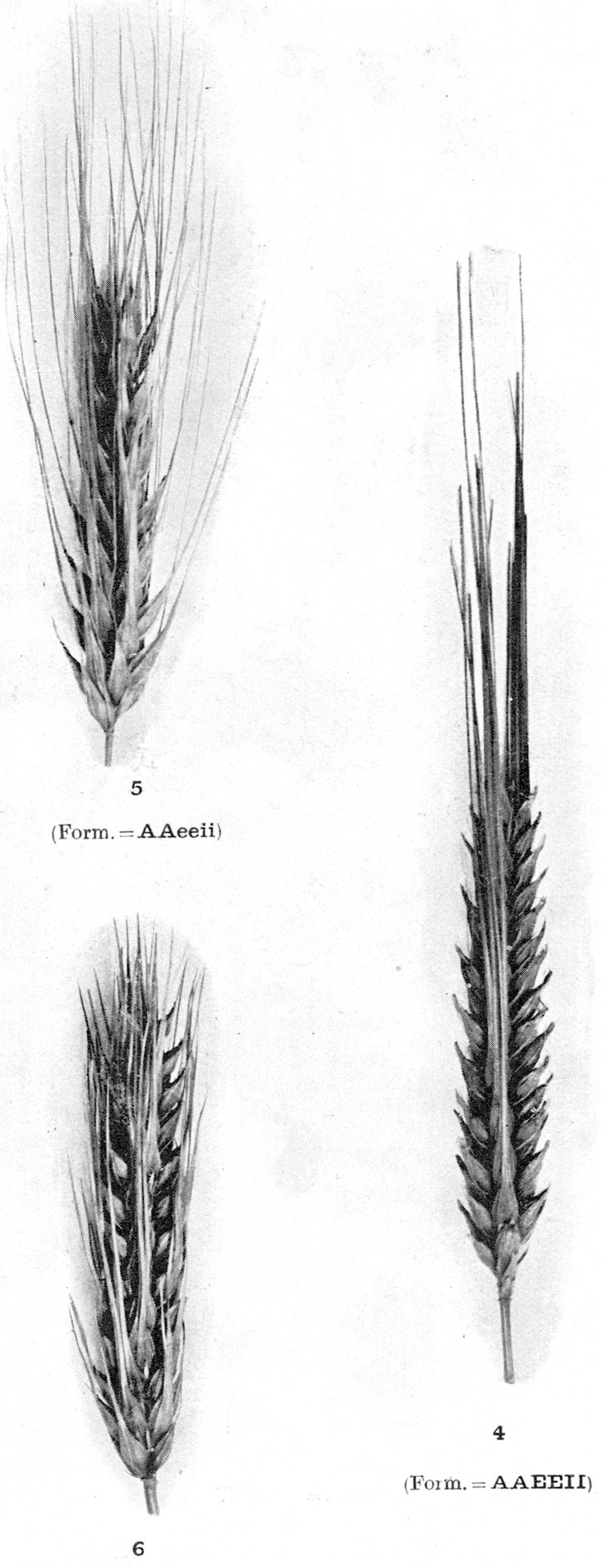

(Form. $=$ aaeeii) 


\title{
Étude génétique sur les arêtes d'une race de l'Orge à six rangs.
}

\author{
Par
}

Se̊itirố Ikeno.

Planches II-III et 1 figure dans le texte.

Une race japonaise de l'Orge à six rangs (Hordeum sativum hexastichum), connu par le nom vulgaire "Kinukawa» (pron. kinoukawa) a tous ses épillets très grêlement aristés (P1. II, fig. 1), bien que les arêtes de ceux appartenant au rang moyen soient quelque peu plus longues que celles appartenant à celui latéral.1)

Pour étudier l'hérédité de ces arêtes j'ai fait un croisement de cette race avec une autre aussi bien à six rangs, appelée vulgairement «Nogenasi ${ }^{2)}$ (pron. noghénasi), où les arêtes font en général défaut; on $\mathrm{y}$ voit cependant très fréquemment la production des arêtes courtes chez un certain nombre des épillets appartenant au rang moyen et il importe de remarquer ici que même dans tels cas les arêtes ne se produisent jamais chez ceux placés à la partie supérieure de l'épi et qu'elles n'ont jamais une longueur suffisamment

1) Le diagramme ci-contre est pour les lecteurs auxquels le mode d'arrangement des épillets chez un épi de l'Orge à six rangs reste inconnu. Il représente la section transversale d'un épi de l'Orge de cette sorte et montre la position des épillets appartenant tant à deux rangs moyens $\left(m_{1}, m_{2}\right)$ qu'à quatre rangs latéraux $\left(l_{1}, l_{2}\right.$, $l_{3}, l_{4}$ ) autour de l'axe $a$. Pour abréger l'expression, nous nommerons ci-dessous les arêtes des épillets appartenant au rang moyen et latéral les «arêtes moyennes» et «celles latérales» respectivement.

2) Noge, arête; nasi, sans.

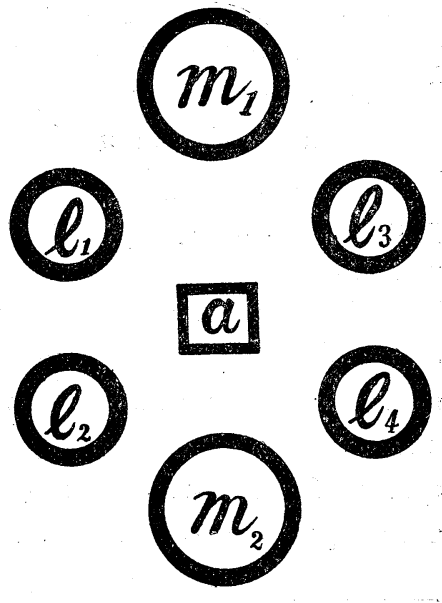

$a=$ axe de l'épi.

$m_{1}, m_{2}=$ rangs moyens.

$l_{1}, l_{2}, l_{3}, l_{4}=$ rangs latéraux. 
grande pour que leur extrémité supérieure puisse atteindre le niveau des épillets placés au sommet de l'épi (P1. II, fig. $2, A$ et $B$.)

Chez les individus de la génération $\mathrm{F}_{1}$, tant Kinukawa $\times$ Nogenasi que Nogenasi $\times$ Kinukawa, les arêtes moyennes sont toujours assez grêles, bien qu'elles soient un peu plus courtes que celles du parent aristé (P1. III, fig. 3). Quant aux arêtes latérales, elles n'y se comportent nécessairement pas toujours le même dans un épi : dans quelques épillets on voit la production des arêtes courtes; dans les autres il n'y en a aucune, mais le sommet de la paillete externe se prolonge très légèrement et est pointu; dans quelques autres non-seulement elle manque entièrement, mais encore le sommet de la paillete reste tout à fait obtus.

La génération $F_{2}$, provenue de celle $F_{1}$ susdite, est marquée par une grande variété d'individus y renfermés. Au premier abord nous avons ceux avec les épis entièrement aristés, ceux avec les épis demi-aristés ${ }^{1)}$ et ceux avec les épis resemblant à ceux de la génération $F_{1}$, où, comme il a été énoncé tout à l'heure, les arêtes latérales se comportent très différemment même chez un épi. La chose devient encore bien plus compliquée, parce que différents individus, soit entièrement aristés, soit demiaristés, ont des arêtes d'une longueur très diverse. Nous en avons eu par exemple entre autres des individus similaires au parent aristé Kinukawa, aussi bien ceux similaires à celui mutique Nogenasi, mais il ne sera pas prudent de déduire leur constitution génétique en se fondant simplement sur leur port externe, car la culture de la génération $\mathrm{F}_{3}$ m'a clairement indiqué que les plantes tout à fait similaires entre elles pendant une génération peuvent se comporter différemment de l'une à l'autre dans la génération suivante.

La culture de la génération $\mathrm{F}_{3}$ nous a fait voir que parmi tous les individus de la génération $\mathrm{F}_{2}$ resemblant au parent aristé Kinukawa, il y a seulement quatre qui restent héréditairement constants et qui peuvent donc être considérés comme la race Kinukawa extraite de 1'hybride Kinukawa $\times$ Nogenasi, et que, en outre, parmi tous les individus de la génération $\mathrm{F}_{2}$ similaires au parent mutique Nogenasi il y a seulement six qui restent constants ci-après. Comme nous avons eu 321 plantes de la génération $\mathrm{F}_{2}$ en tout et d'ailleurs nous en avons eu ainsi quatre Kinukawa et six Nogenasi qui restent constants ci-après, on verra qu'il est très vraisemblable que nous avons ici affaire à un cas

1) Les épis avec toutes les deux sortes d'arêtes, tant moyennes que latérales, sont appelés ici «entièrement aristés 》 et ceux seulement avec les arêtes moyennes «demiaristés.» 
de nature trihybride ou 《trifactorielle 》, car le rapport 4 ou 6 , c'est-àdire 5 en moyenne pour 321 est précisément égal à celui 1 pour 64 . En effet, les résultats de tous mes expériences de culture ont confirmé ma supposition et $\mathrm{m}$ 'a conduit à la conclusion énoncée ci-dessous. ${ }^{1)}$

Nous avons ici trois facteurs ou gènes que je représente par les lettres $\mathbf{A}, \mathbf{E}$ et $\mathbf{I}$ respectivement. Et remarquons tout d'abord que même en absence de ces trois facteurs (c'est-à-dire chez l'individu aaeeii) on voit le développement des deux sortes des arêtes (moyennes et latérales) très courtes (Pl. III, fig. 6). En présence de l'un des deux facteurs $\bar{A}$ ou $\mathbf{E}$ les arêtes, tant moyennes que latérales, sont pourtant plus longues qu'en leur absence (Pl. III, fig. 5) et en présence simultanée de tous les deux elles sont très grêles (Pl. II, fig. 1), AAEEii étant en effet le parent aristé Kinukawa. L'action du troisième facteur $\mathbf{I}$, qu'on peut appeler celui d'empêchement, est à supprimer entièrement le développement des arêtes latérales, aussi par exemple l'individu AAEEII est-il marqué par le développement de longues arêtes moyennes et par l'absence entière de celles latérales (P1. III, fig. 4). Chez l'individu aaeeII, c'est-à-dire chez le parent mutique Nogenasi, on voit que le facteur I non-seulement supprime le développement des arêtes latérales, mais encore il empêche celui de celles moyennes en entier ou presque en entier (P1. II, fig. 2, $A$ et $B$ ).

L'hybridation de Kinukawa (AAEEii) par Nogenasi (aaeeII) ou la réciproque donne par conséquent AaEeli (Pl. III, fig. 3). Le développement des arêtes moyennes plus courtes dans cet hybride que dans le parent Kinukawa, aussi bien la diversité de la conduite des arêtes latérales daus cette génération énoncée ci-dessus, seront très facile à comprendre, vu la présence des facteurs $\mathbf{A}, \mathbf{E}$ et $\mathbf{I}$ en état hétérozygote.

$\mathrm{Si}$ nous nous basons sur la théorie, il faut que nous aurons en $\mathrm{F}_{2}$ les plantes appartement à trois classes suivantes, le chiffre inclus entre les deux parenthèses indiquant le nombre des pieds de chaque plante pour 64:-

$1^{\circ}$ Plantes entièrement aristées, c'est-à-dire ne renfermant aucun facteur I-ĀAEEii (=Kinukawa)(1), A AEeii (2), AaEEii (2), AaEeii (4), ÁAeeii (1), Aaeeii (2), aaEEii (1), aaEeii (2), aaeeii (1)-en tout 16 pour 64 ;

$2^{\circ}$ Ceux demi-aristés et mutiques, renfermant le facteur $I$ en état homozygote-AAEEII (1), AAEEII (2), AaEEII (2), AaEeII (4), AAeeII (1), AaeeII (2), aaEEII (1), aaEeII (2), aaeeII (=Nogenasi) (1)-en tout 16 pour 64 ;

1) De tous les expériences antérieurs concernant les arêtes de l'Orge je nom-. merai ici simplement ceux de Tschermak (Fruwirth, Die Züchtung der landw. Kulturpflanzen, Bd. 4. 2. Aufl., 1910, p. 311). 
[Vol. XXXI, No. 370 .

$3^{\circ}$ Ceux renfermant le facteur I en état hétérozygote-AAEEIi (2), AAEeli (4); AaEEli (4), AaEeli (8), AAeeli (2), Aaeeli (4), aaEEIi (2), aaEeli (4), aaeeli (2)-en tout 32 pour 64 .

Le problème, à laquelle de ces trois classes énumérées ci-dessus appartiendra-t-il un certain individu de la génération $F_{2}$, peut être sûrement résolu au moyen de l'examen de leur progéniture, c'est-à-dire de la génération $F_{3}$. Car il est évident que les individus de la classe I et II donneront naissance alors exclusivement à ceux entièrement aristés et demi-aristés respectivement, tandis que ceux de la classe III qui renferment le facteur I en état hétérozygote (c'est-à-dire Ii) engendreront alors en conformité à la loi mendélienne les deux sortes de la progéniture, tant entièrement aristées que demi-aristées. ${ }^{1)}$

Voici les résultats de ma culture de la génération $\mathrm{F}_{3}$ :-

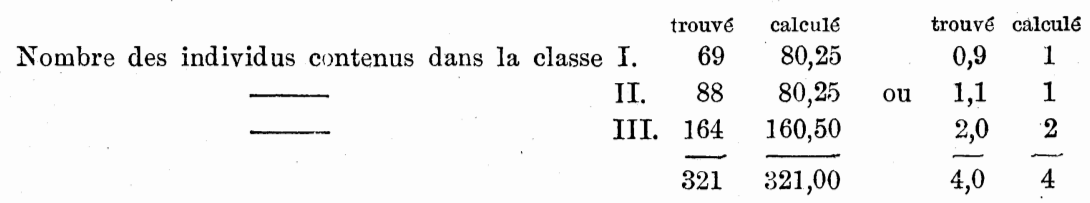

Cela confirme presque entièrement ce que l'on a prévu théoriquement.

De l'inspection de l'énumération des individus $F_{2}$ énoncés ci-devant on verra que d'après la théorie nous faudrons avoir dans chacune des deux classes $I$ et II pour 16 individus 4 qui resteront tout à fait constants dans la génération suivante (c'est-à-dire $1 / 4$ de tous les individus) et qui sont AAEEii, AAeeii, aaEEii, aaeeii et AAEEII, AAeeII, aaEEII, aaeeII respectivement et que dans la classe III il n'y a aucun qui restera constant, car elle est marquée par la présence du facteur $I$ en état hétérozygote. En effet, voici les résultats de mes expériences concernant cette question:-
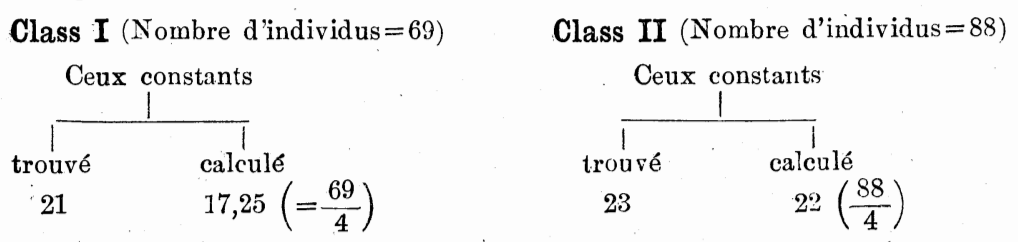

Class III (Nombre d'individus $=164$ ).

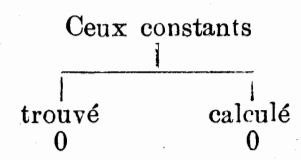

1) Par exemple AAEEIi donne outre AAEEIi, AAEEII (demi-aristé) et AAEEii (entièrement aristé). 
Les résultats de mes expériences se résument done comme suivants :nous avons affaire à trois facteurs $\mathbf{A}, \mathbf{E}$ et $\mathbf{I}$, dont les deux premiers sont renfermés dans le parent aristé Kinukawa et le dernier se trouve dans celui mutique Nogenasi. Même sans aucun de ces trois facteurs les arêtes moyenries et latérales courtes peuvent être développées, mais en présence de l'un des deux facteurs, soit $\mathbf{A}$, soit $\mathbf{E}$, les arêtes sont plus longues et en présence simultanée de tous les deux elles peuvent croître jusqu'à une longueur beaucoup plus grande. I, le facteur d'empêchement, en état homozygote, agit par supprimer la production des arêtes latérales, mais il n'est pas suffisamment puissant pour pouvoir empêcher absolument le développement des arêtes moyennes, d'où l'on pourra comprendre la raison pourquoi même dans le parent mutique Nogenasi (aaeeII) quelques arêtes moyennes courtes peuvent-elles se produire. Quand le facteur d'empêchement est en état hétérozygote, nous voyons que la conduite des arêtes latérales est très variable même dans un épi. La grande diversité de longueur des arêtes est principalement due à la combinaison diverse des deux facteurs $\mathbf{A}$ et $\mathbf{E}$.

Voilà le résumé de mes expériences et il est tout naturel que tous les détails de mes recherches seront consignés dans un mémoire ultérieur complet.

\section{Explication des Planches.}

Toutes les figures sont les photographies faites presque en grandeur naturelle.

\section{Planche II.}

Fig. 1. Parent aristé, Kinukawa. Formule=AAEEii. Vu d'un côté un peu obliquement, c'est-à-dire les deux rangs latéraux d'un de ses côtés placés en devant.

Fig. 2. Parent mutique, Nogenasi. Formule=aaeeII. $A$. On n'y voit aucune arête. $\mathrm{Vu}$ de face, c'est-à-dire un des deux rangs moyens en devant. $B$. On y voit un certain nombre des épillets moyens courtement aristés. Vu du côté, c'est-à-dire les deux rangs latéraux d'un côté en avant.

\section{Planche III.}

Fig. 3. Individu $\mathrm{F}_{1}$. Formule=AaEeIi. Vu de face, c'est-à-dire un de ses deux rangs moyens en devant. On y voit les arêtes courtes appartenant aux épillets des rangs latéranx.

Fig. 4. Individu AAEEII avec les arêtes moyennes longues sans aucune arête latérale. $\mathrm{Vu}$ de face.

Fig. 5. Individu AAeeii avec les arêtes moyennes et latérales, qui ne sont pas très longues. Vu de face un pen obliquement.

Fig. 6. Individu aaeeii avec les arêtes plus courtes que chez Fig. 5, tant moyennes que latérales. Vu de face un peu obliquement. 